\title{
Simulation of the preparation and face drilling processes with laser scanning and automated marking of the drilling grid
}

\author{
Evgeniya $\mathrm{V}_{\text {olkova }}{ }^{1,}{ }^{*}$, Aleksey Druzhinin ${ }^{1}$, Roman $\mathrm{K}$ uzminykh $^{1}$, and Vladimir Poluzadov ${ }^{1}$ \\ ${ }^{1}$ U ral State M ining University, 620144, 30, Kuibyshev st., Ekaterinburg, Russia
}

\begin{abstract}
The article discusses the methods of calculating the drilling and blasting scheme and constructing a drilling grid, manual and automatic calculation options are compared. A method for automatically constructing a drilling grid based on laser scanning is proposed. M oreover, the proposed method can be implemented using cheap equipment - a laser rangefinder and an A rduino microcomputer. Based on the data of the laser rangefinder with openCV and SciPy libraries, a polygonal 3D model of the face is built. The transfer of the drilling grid to the 3D model is implemented using the bilinear interpolation algorithm. The constructed polygonal model can be improved by making changes to the construction algorithm, since it is developed by the authors and can be further developed. The simulation model is created in Anylogic software and shows the drilling process taking into account the previously calculated drilling pattern. The proposed models can be used as a basis for further research and software development.
\end{abstract}

\section{Introduction}

Nowadays, there are many problems in drilling-and-blasting for horizontal tunnelling, such as low accuracy of marking the drilling grid, low accuracy of pointing the jumbo rig tool, errors in calculating and marking of the drilling grid, and, as a result, low speed of overall mining process. In most underground mines in Russia and the CIS countries, the process of making up a drilling grid and the drilling process itself proceeds to a methodology that is quite costly in terms of man-hours: first, the mining foreman scans the mining face, then calculates the drilling pattern and generates a drilling-and-blasting certificate, then marks the drilling grid ant the face [1]. Despite the availability of modern means of applying the drilling grid, it is often done manually using paint. After the grid is marked, the operator of the jumbo rig can start the drilling process. The final accuracy of the face drilling process with this method is rather poor, since the human factor plays a role at all stages of the process, from calculating the drilling pattern to pointing the jumbo tool and drilling the blast-holes.

This problem can be solved through the automated jumbo rig usage, which are equipped with software and hardware for scanning the face, modeling and the processing the drilling grid, remote control of the jumbo rig tool, as well as data mining and analyzing [2]. However, not all mines are equipped with such complexes, and retooling can be extremely expensive.

*Corresponding author: evgeniya.volkova@m.ursmu.ru 


\section{Materials and Methods}

As part of the solution to the problem of increasing the speed, accuracy and efficiency of drilling and blasting, the authors chose a simulation method that is used for the weaknesses of the current approach identification, as well as to conduct experiments on imitation models of real objects. This article discusses the simulation of the processes of calculating a drilling grid and drilling, without taking into account the processes of explosives loading, blasting and subsequent steps (cleaning the face, anchoring). Modelling of these stages is planned to be carried out after the end of the current development.

One of the possible solutions is a computer calculation of the drilling and blasting certificate data, according to already existing generally accepted formulas. It is also an important fact that the automatic system will consider this many times faster, without additional resources.

Another approach is to use software packages for calculating, for example, Micromine or Mineframe. In this case, the human factor is also excluded in the calculation, however, the calculation algorithms used in this software are private, and therefore, it is impossible to analyze the influence of certain factors on the final calculation.

For example, consider one of the established methods for calculating the drilling grid. According to the generally accepted approach, the number of inclined blast-holes is determined by the formula (1):

$$
N=\left(q * S_{d r}\right) / \gamma,
$$

where $N$ is for corresponding number of blast-holes, $q$ is for specific explosive charge, $\mathrm{S}_{\mathrm{dr}}$ is for draft cross-sectional area and $\gamma$ this is for the weight quantity of explosive per $1 \mathrm{~m}$ hole, $\mathrm{kg} / \mathrm{m}$. Explosive charge is determined by formula (2):

$$
q=\left(0,1 * f * f_{1} * v\right) / e \text {. }
$$

where $q$ is for specific explosive charge, $f$ is for the of rock strength coefficient according to Protodyakonov scale, $f_{l}$ is for the rock structure coefficient, $v$ is for the rock clamp coefficient and $e$ is for the explosive charge efficiency coefficient.

The main drawback of this approach is the large number of coefficients in the calculations, which leads to different results depending on the degree of rounding. On the other hand, this method of calculating the number of blast-holes has not changed for years, is clearly verified and has proved to be quite effective in practice. However, the effectiveness of the result often depends on the experience of the specialist performing the calculation.

Figure 1 shows an example of calculating a blast passport in Micromine software. The calculation result based on formulas (1-2), depending on rounding, is 17-21 blasting-holes; in Micromine, as a result of calculations, the drilling grid consists of 17 blasting-holes, which shows approximately the same values. However, the drilling grid of 21 blast-holes differs significantly from the grid of 17 blast-holes, and in the case of manual calculation, the responsibility for the choice lies with the specialist.

In general, we can say that usage of the considered calculation method can be applied to further develop a system for automating the drilling process, but needs to increase accuracy by introducing additional parameters into the calculation. 


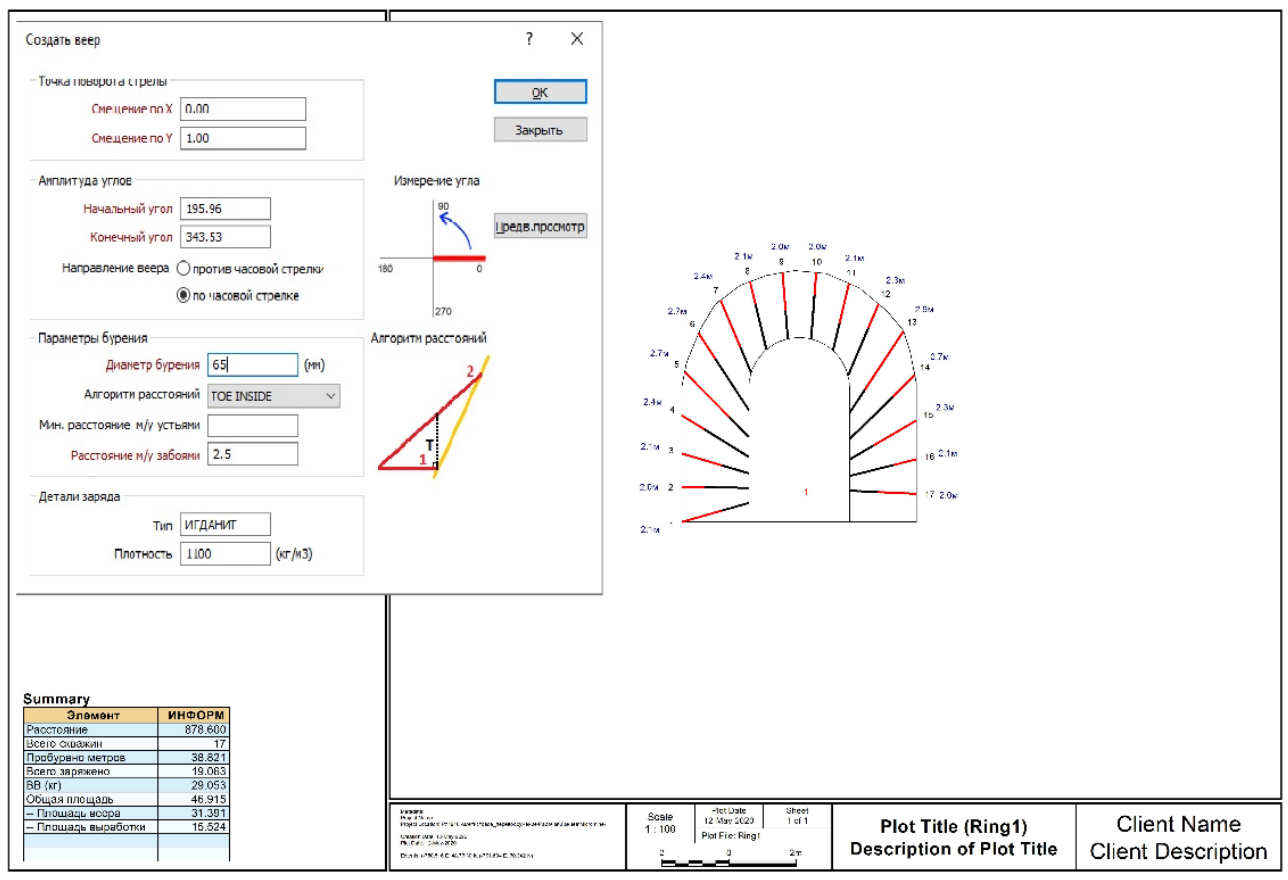

Fig. 1. B lasting-and-drilling calculation with M icromine software

However, in addition to the problem of calculating the drilling-and-blasting certificate, there is also the problem of the accuracy of applying the resulting drilling grid to the surface of the mine, as well as the accuracy of the drilling itself on the applied grid. One of the approaches to improving the quality of drilling blasting-holes on the surface is the usage of laser scanning and the subsequent adaptation of the drilling pattern in accordance with the real geometry of the face [3-7]. Laser scanning data after drilling process can also be useful for spatial juxtaposition of blasting-holes with drifts and drilling quality assessment. Expensive drilling rigs have a pre-installed laser scanning station on board, but with less modern machines, a scanning device is required. A cheap solution can be a single laser on a rotary joint, which you can provide a 3D model sufficient for accuracy calculations.

For the experiment, the authors used a HIREED laser rangefinder without a rotary hinge connected to the Arduino microcomputer, and the polygonal model shown in Figure 2 was constructed from the data obtained. Polygonal model is performed with Python openCV and SciPy libraries based on the laser rangefinder data log.

To build a drilling grid on a three-dimensional model, there are several options: you can use the existing mlab library algorithms (MathLab) or independently implement the bilinear interpolation algorithm [8]. The second option is preferred, as it will allow to make adjustments to the algorithm to achieve the required parameters. The resulting $3 \mathrm{D}$ drilling grid can be used for automatically marking of a drilling blasting-hole point using a laser.

The idea of automatically marking a drilling point is to use a laser to highlight the corresponding face point, so that the drilling rig operator can accurately point the working tool at it. Scan ability will provide feedback and control the quality of drilling. 


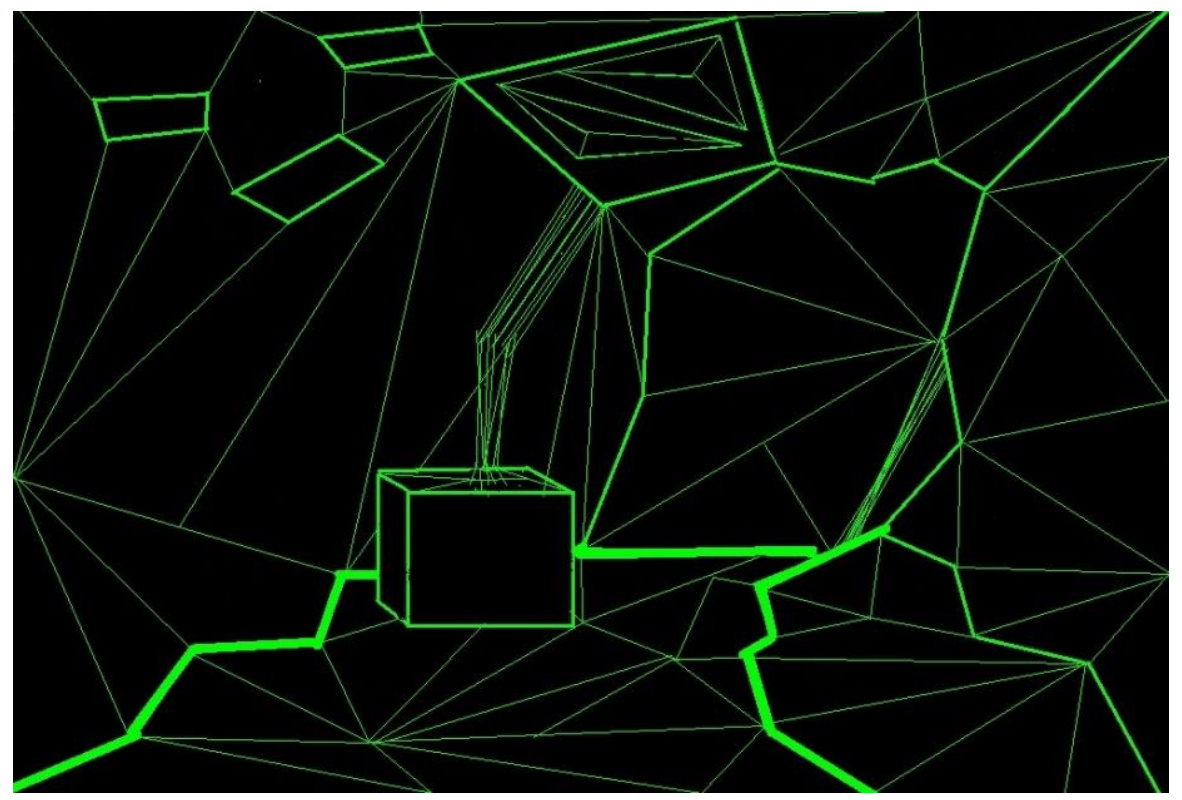

Fig. 2. Polygonal face model

The next step of preparation and drilling modelling is the creation of a simulation model of the entire working cycle in the Anylogic software. The result of simulation is presented with Figure 3.

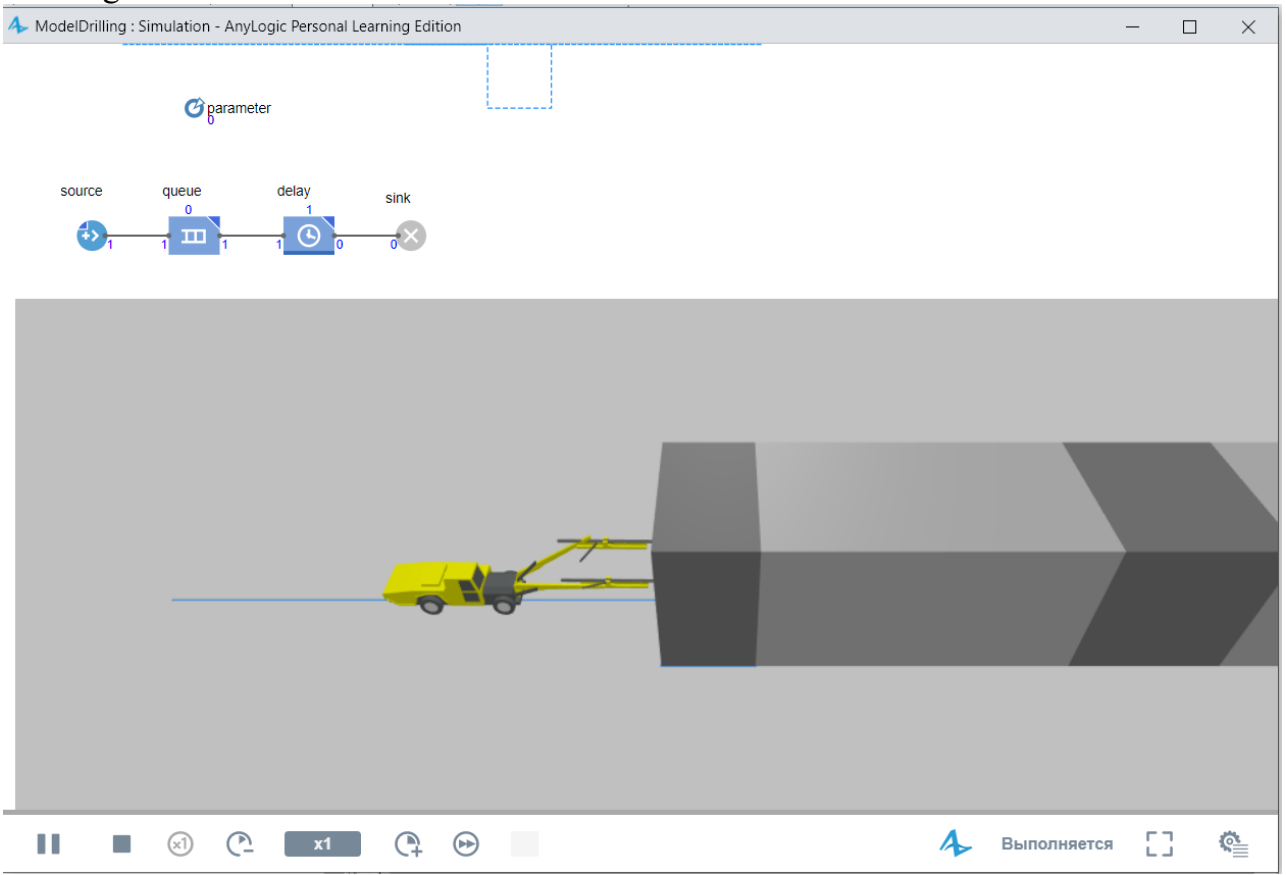

Fig. 3. D rilling simulation in A nylogic software

On Figure 3, drilling process is in progress and the fist blast-hole is performed (it is agent number one in source block). Modeling of preparation processes remains to be added to this model, which is planned to be done. 


\section{Results and Discussion}

As a result of the study, comparisons of drilling grid design methods were carried out and a technique was selected for the implementation of a system for automatically applying a grid to the face. An experiment was also conducted to build a 3D model of the face using laser scanning based on a laser rangefinder and an algorithm for constructing a polygonal model. The low accuracy of the polygonal model is due to the small number of measurements, as well as the use of the simplest algorithm for constructing a polygonal $3 \mathrm{~d}$ model without smoothing methods and precision improving. In the future, it is planned to refine the algorithm to increase the accuracy of the model, which is the topic for further research. Another thing that have to be made is implementation of the bilinear interpolation algorithm on Python to superimpose a drilling grid on the 3D face model.

Simulation of the drilling process is implemented in software Anylogic. At the moment, the simulation shows the drilling process based on the designed drilling pattern. It is planned to add to the simulation the building of a drilling grid and positioning the drill tool processes.

\section{Conclusion}

The approaches to the calculation and construction of the drilling grid, considered in the article, can be applied for further research in the field of automating the process of drilling blast-holes with horizontal penetration.

The constructed simulation can be used to conduct computational and simulation experiments, and serve as the basis for the development of algorithms, more accurate models and software. Based on the proposed methods, it is planned to create a prototype hardware and software complex for automatic scanning of the mining face and positioning of the drill under the drilling point.

\section{Acknowledgements}

The studies are carried out as part of the implementation of the contract under the grant UMNIK No. 14207GU / 2019 dated 06/21/2019.

\section{References}

1. K. Karginov, A. Lipovoj, T. Teziev, D. Rjabov, GIA B, 8 (2001)

2. J. Navarro, J.A. Sanchidrián, P. Segarra, R. Castedo, E. Costamagna, L.M. López, Tun. and Undergr. Sp. Tech., 82, 504-516 (2018)

3. S. Milanović, L. K ricak, M. Negovanović, N. Simic, J. Marković, Podzemni radovi, 2019, 77-89 (2019)

4. B. Rymarchuk, 0 . Shepel, W ays of increase of efficiency of drilling-and-blasting, E3S W eb of Conferences, 166, 03001 (2020) doi 10.1051/e3sconf/202016603001

5. S. M ukhtar, GIM International, 32, 22-25 (2018)

6. S. Grehl, M. Sastuba, M . Donner, M . Ferber, F. Schreiter, H. M ischo, B. J ung, T owards virtualization of underground mines using mobile robots - from 3D scans to virtual mines (2015)

7. D. Huber, N. Vandapel, Automatic 3D Underground Mine Mapping, doi 10.1007/10991459_48 (2006)

8. K.H. Kim, P.S. Shim, S. Shinun, Atmosphere, 10, 123 (2019) doi 10.3390/atmos10030123. 